\title{
Dangerous Degree Evaluation of Mine Debris Flow Based on the Immune Genetic Neural Network ${ }^{(1)}$
}

\author{
Xicheng Xue ${ }^{\mathrm{a}}$, Jisong $\mathrm{Bi}^{\mathrm{a}}$, Lingling Chen ${ }^{\mathrm{a}}$ \\ ${ }^{a}$ College of Geology and Environment, Xi'an University of Science and Technology, Xi'an, China
}

\begin{abstract}
Taking the western Qinling Mountain, in the southern Shaanxi Province of china, as an example, based upon comprehensive analysis of geological data for 20 debris flow gullies, the author has put forward a series of indices system and has developed the immune genetic neural network system, which can quantitatively evaluate the dangerous degree of mine debris flow. This software system manage initial data through Access's data-base technology, and determine and optimize the hidden layer network by immune genetic algorithm, as well as achieve the dangerous degree evaluation of mine debris flow by virtue of artificial neural network which has been successfully trained. The calculating results of mine debris flow examples testify that this method is reliable and can accurately evaluate the dangerous degree of mine debris flow. These evaluation results have some important instructive significance for the disaster prevention and reduction of mine debris flow.
\end{abstract}

Index Terms: Qinling Mountain; Mine Debris Flow; Dangerous Degree; Indices System; Immune Genetic Algorithm; Artificial Neural Network

(C) 2012 Published by MECS Publisher. Selection and/or peer review under responsibility of the International Conference on E-Business System and Education Technology

\section{Introduction}

The western Qinling is located in the source of Jialing River and Han River, which is rich in metal mineral resources, such as $\mathrm{Pb}, \mathrm{Zn}, \mathrm{Au}$ and so on. These metals are concentrated in mineral accumulation area such as Fengxian, Taibai ect.. Since the reform and open, various regions have been developing the mining economy positively by the right of their resource superiority. The mining development has made a significant contribution for the development of the local economy as well as the social progress. However, because of the high-strength exploit of mineral resources, disasters caused by mining activities such as the strong disturbance of geological environment, the recklessly piling of mine spoil, waste rock and tailings and mine debris flow

${ }^{(1)}$ The Subsidy of Shaanxi Nature Science Fund Project (SJ08-ZT08-3) 
occur frequently [1]. In August 1981, affected immediately by the strong rainstorm, large areas of debris flow occurred in western Qinling. The debris flow whose main source is mine spoil buried a train of Honghuapu Station. And the bridges and roads were blocked and destroyed by detritus. The traffic was suspended for 60 days. The national economy suffered heavy losses [2]. On September $18^{\text {th }}, 2001$, after a heavy rain, a mine debris flow occurred abruptly in qiantongshan mining area. The mountain slumping stuff in the upper reaches of the cleugh and the mine spoil poured down. As a result, the transport vehicles were buried, what's more, the traffic of the mining area was suspended, which caused heavy losses. So, the dangerous degree evaluation of mine debris flow should be put into effect as soon as possible in order to instruct the prevention and reduction of disaster of mine debris flow scientifically.

\section{The Limits of Traditional Mathematical Evaluation Models}

There are many factors influencing the dangerous degree of mine debris flow. Assessing its dangerous degree scientifically is the important foundation of the planning and engineering design for the management of mine debris flow. For the past few years, a lot of scholars have been proceeding researches on mine debris flow from different perspectives. And they have obtained plentiful and substantial achievement [3-7]. From the traditional torrent classification, quantitative discrimination act to the following fuzzy comprehensive evaluation method, mathematical methods are increasingly used in the fields of mine debris flow risk. While the superiority of those mathematical methods mentioned above is fully displayed, their disadvantages are also exposed in practice, which has attracted increasing attention. Methods which were used earlier such as the torrent classification and quantitative discrimination act are easy to operate, but the formulas themselves are in the status of semiquantitative "man-made scoring" in which the human experience and subjective effects account for the most part. Fuzzy mathematics has a strong ability of data expression. It can take different index weights into account and carry out comprehensive evaluation on multi-factors. But in practice, it is often difficult to determine the membership function and weight sets.

\section{The Present of Evaluation Method Based on the Immune Genetic Neural Network}

The mine debris flow risk can be measured by a dangerous degree which has a probability concept. Here the dangerous degree is valued in the closed interval of $[0,1]$. With the development of artificial intelligence and object-oriented programming technology, it will be an inevitable trend to evaluate the mine debris flow risk using theory related to artificial intelligence. At present, artificial neural network model is used widely because of its advantages of self-organization, self-learning, concertedness and high precision. Compared with those traditional mathematical methods which need to determine membership function and weight sets artificially and don't have self-learning, association and memory function, the artificial neural network model can solve these problems perfectly. But the artificial neural network model still has its disadvantages. The determination of its hidden layer structure, the number of neurons, the initial threshold and the initial connection weight value is faced with the problem of global optimization. Given this, it lays the foundation for the train of the artificial neural network model to attempt to adopt the genetic algorithm mathematical model that based on immune theory or IGA Model. Due to the immunological memory and self-regulation of IGA Model, the global search ability of genetic algorithm can be enhanced, and thus can avoid trapping into local solution. IGA Model also has the function of maintaining the diversity of antibodies. The function can enhance the global search ability, avoid premature convergence and insure a rapid converge to the global optimum solution.

\section{The Fundamental of BP Neural Network}

The artificial neural network is a kind of nonlinear mathematical method based on the theory of imitating the impulse conduction of human cranial nerve. It is a large-scale complex net system composed of numerous neurons by a fixed topological structure. It is used to imitate the structure and action of human cranial nerve 
network [8]. The artificial neural network can learn knowledge automatically from the experience of experts as long as provided sufficient representative learning samples. At the same time, it can solve problems by the mean of summarizing judge rules and modify evaluation mathematical model by itself. In the light of the differences of network function, structure and learning style, the artificial neural network model can be divided into several kinds of models, of which a more mature one is multi-layer error back propagation neural network(BP neural network for short). This kind of model consists of input layer, hidden layer and output layer, and they connect each other by full mesh (Fig. 1).

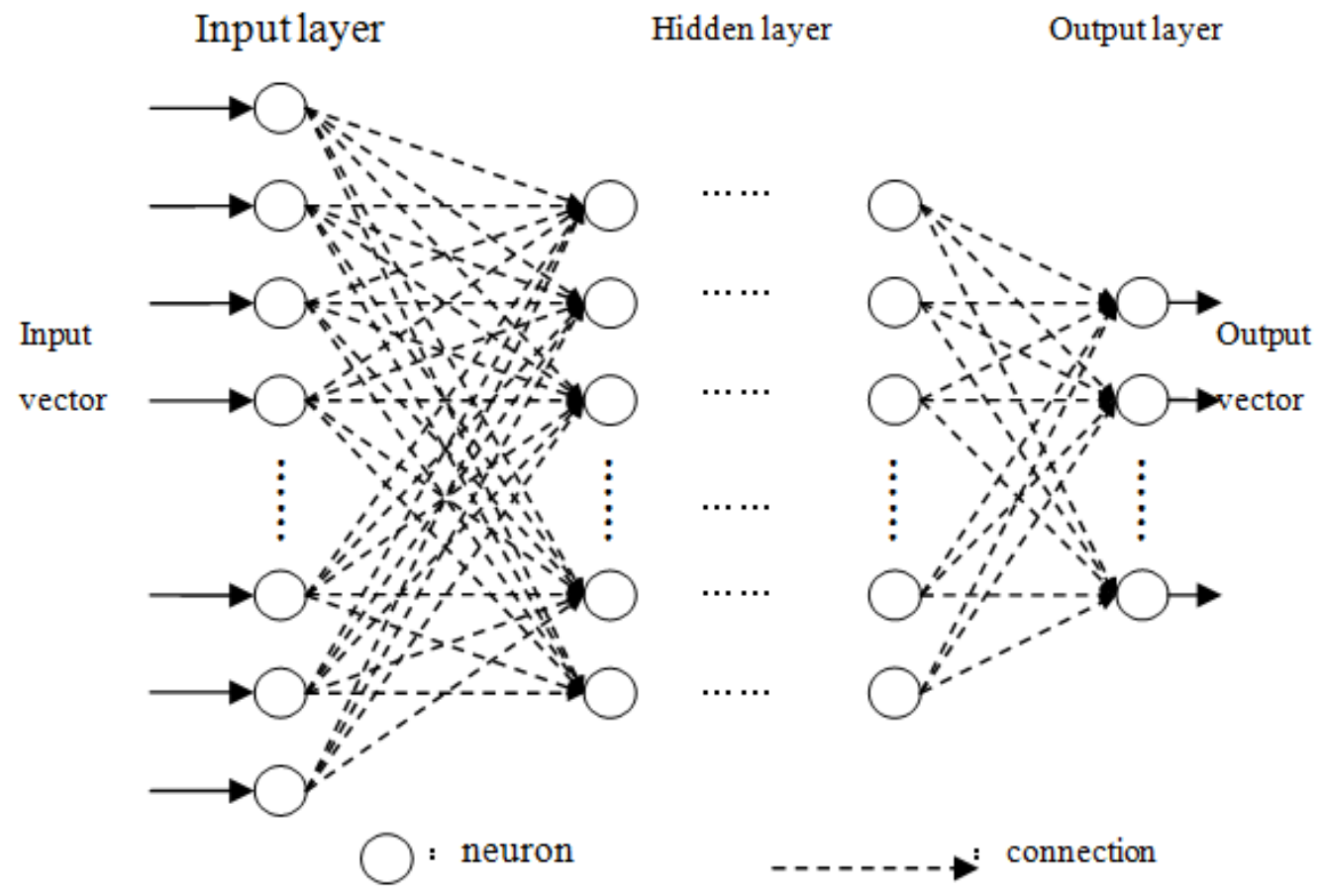

Fig. 1. The structure sketch map of neural network

\section{The Fundamental of Immunity Genetic Algorithm}

Genetic Algorithm is a kind of random search algorithm taking example by natural selection and nature genetics mechanism in biosphere. It takes the objective function of practical problems to be solved corresponding to antigen while the solution of problems is corresponding to antibody. It applies to the complex and nonlinear problems those can't be solved by traditional search algorithm. However, in practice, there are some problems to be discussed further [9-10]. For example, for the monotonic functions or unimodal functions, they approach the optimal value rapidly at the beginning while converge slowly nearby the optimal value. For the optimization problems of multimodal functions, they always converge to local extremum rapidly. While the genetic algorithm (Immunity Genetic Algorithm, or IGA for short), based on immune theory put forward in this paper, is an improvement of traditional genetic algorithm. This complex and new algorithm can improve the drawback of premature convergence and enhance the global and local search ability of genetic algorithm.

The specific process of immunity genetic algorithm is devised according to the certain chromosome coding scheme and immune operators, as is shown in Fig. 2. The objective function and all kinds of evaluation indexes 
are input at first to act as antigens of immunity genetic algorithm. Then a set of initial antibodies (corresponding to candidate solutions) are produced randomly. For the secondary response, it needs the help of the memory function of immune mechanism. Parts of the initial antigens are obtained through mnemon. The antibodies in mnemon have good fitness and population distribution so that the convergence rate can be enhanced. And then the fitness of every generation of antibodies are calculated with crossover and mutation carried out on these antibodies. Afterwards, generate the next generation of antibody groups by updating strategy based on the groups of antibody concentration and affinity. End the whole algorithm till the end condition is satisfied. Finally, the results are substituted into BP neural network to carry out the network training and evaluation.

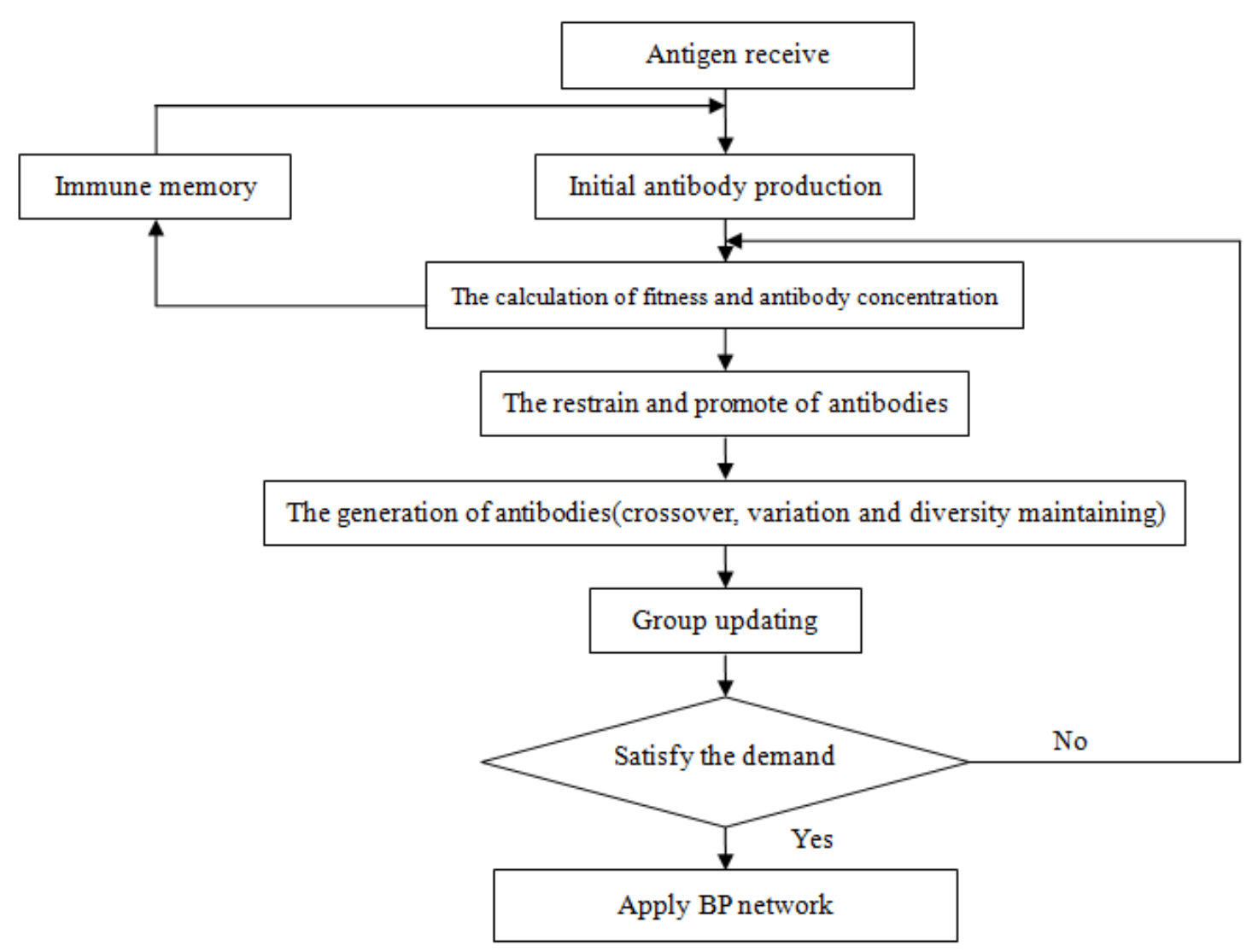

Fig. 2. The flow chart of immunity genetic algorithm

\section{Dangerous Degree Evaluation of Mine Debris Flow Based on the Immune Genetic Neural Network}

\subsection{The Treatment Process of Software System}

After the basic parameters of genetic algorithm are set up in software system, the program module of immunity genetic algorithm is used to obtain global optimized BP neural network hidden layer structure and network parameters through complicated iterative computations. 
The results of immunity genetic algorithm show that for the certain training sample data and number of input and output neurons, the optimal hidden layer structure is as follows: the hidden layer is 1 and the number of hidden layer neurons is 8 . It can be confirmed that the type of the optimize structure of BP neural network is 33-8-3.The immunity genetic algorithm and artificial neural network mathematical model were combined together. The immunity genetic algorithm was used to optimize the hidden layer structure and network parameters of BP neural network. Then 7269 trains were made on BP neural network using "sample models" of mine debris flow with given dangerous degree, and the network intelligence evaluation of the mine debris flow risk were realized in the end(Fig. 3).

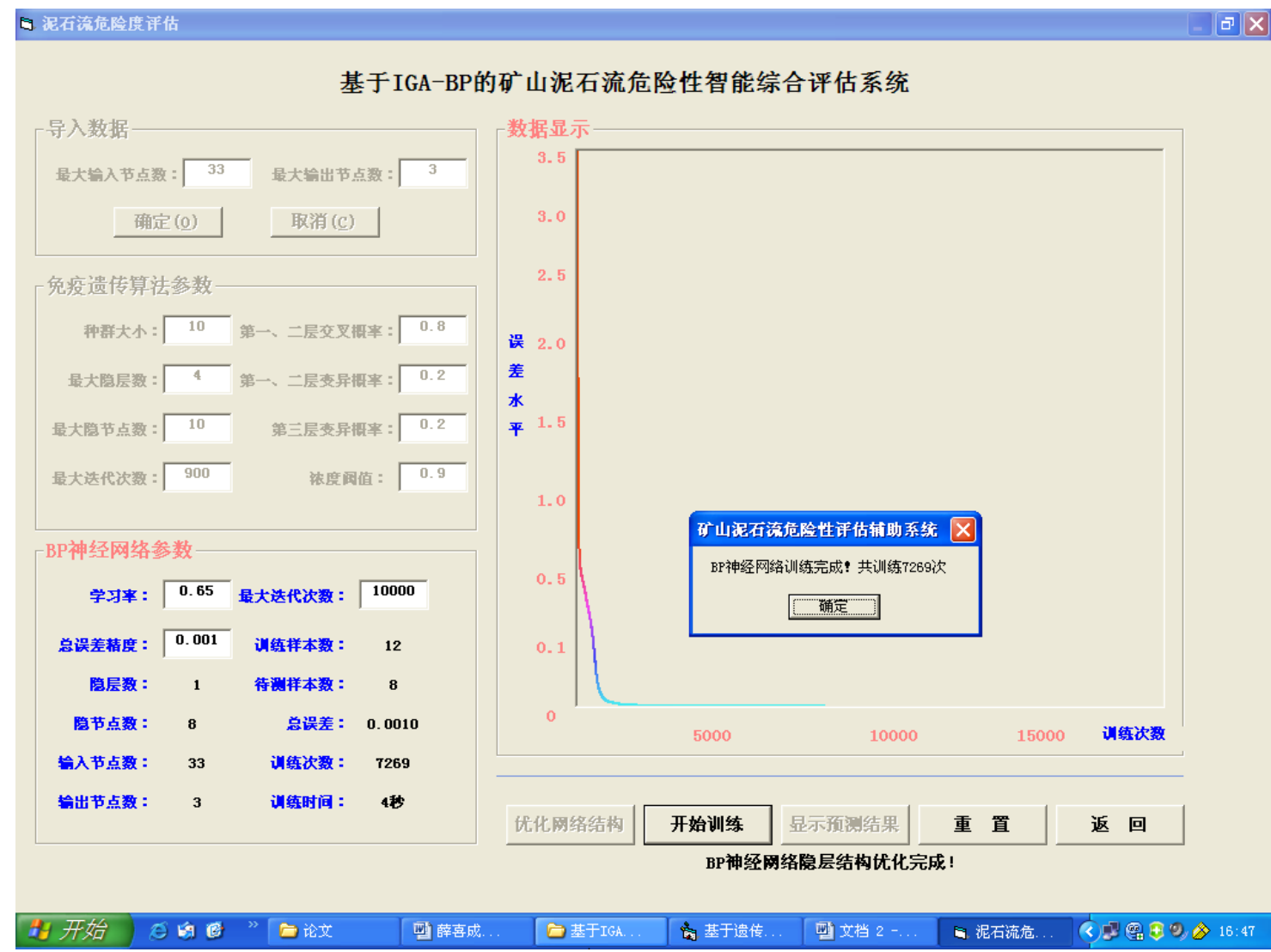

Fig. 3. Main interface of software system

\subsection{The Distinction examination of BP neural network training samples}

After the training of neural network mentioned above, neural network models which had been trained were used to do distinction examination on 24 training sample data. As a result, 3 output neuron values, absolute error, relative error and the types of dangerous degree of mine debris flow were obtained (TABLE 1). The results show that for the net expectant output values of training samples, the average absolute error of the whole training samples is 0.015 , the average relative error is only $5.1 \%$, and no erroneous judgement occurred. It indicates that the fitness of BP intelligent comprehensive evaluation models to the evaluation of training samples is high. So it can be used to make intelligent comprehensive evaluation to the mine debris flow risk. 
Table 1. Distinction examination effect of BP neural network training sample

\begin{tabular}{|c|c|c|c|c|c|c|c|}
\hline Number & Name & Neuron 1 & Neuron 2 & Neuron 3 & $\begin{array}{c}\text { Absolute } \\
\text { error }\end{array}$ & $\begin{array}{c}\text { Relative } \\
\text { error }\end{array}$ & $\begin{array}{l}\text { Dangerous } \\
\text { degree }\end{array}$ \\
\hline N1 & Daheigou & 0.073 & 0.952 & 0.006 & 0.048 & $4.8 \%$ & Subsidiary risk \\
\hline $\mathrm{N} 2$ & Jiashangou & 0.073 & 0.975 & 0.006 & 0.025 & $2.5 \%$ & Subsidiary risk \\
\hline N3 & Zhaizigou & 0.016 & 0.970 & 0.011 & 0.030 & $3.0 \%$ & Subsidiary risk \\
\hline N4 & Shuijingwan & 0.057 & 0.025 & 0.959 & 0.041 & $4.1 \%$ & Risk \\
\hline N5 & Guanmengou & 0.073 & 0.031 & 0.904 & 0.096 & $9.6 \%$ & Risk \\
\hline N6 & Yinmusigou & 0.038 & 0.019 & 0.930 & 0.070 & $7.0 \%$ & Risk \\
\hline N7 & baguagou & 0.031 & 0.962 & 0.015 & 0.038 & $3.8 \%$ & Subsidiary risk \\
\hline N8 & Hetaogou & 0.053 & 0.011 & 0.935 & 0.065 & $6.5 \%$ & Risk \\
\hline N9 & Momengou & 0.027 & 0.948 & 0.065 & 0.052 & $5.2 \%$ & Subsidiary risk \\
\hline N10 & Mahuanggou & 0.025 & 0.937 & 0.041 & 0.063 & $6.3 \%$ & Subsidiary risk \\
\hline N11 & Changgou & 0.065 & 0.897 & 0.010 & 0.103 & $10.3 \%$ & Subsidiary risk \\
\hline N12 & Erlihe & 0.003 & 0.034 & 0.921 & 0.079 & $7.9 \%$ & Risk \\
\hline N13 & Xiaodongwan & 0.001 & 0.936 & 0.036 & 0.064 & $6.4 \%$ & Subsidiary risk \\
\hline N14 & Lishugou & 0.024 & 0.075 & 0.958 & 0.042 & $4.2 \%$ & Risk \\
\hline N15 & Shuijinggou & 0.056 & 0.042 & 0.976 & 0.024 & $2.4 \%$ & Risk \\
\hline N16 & Guoansi & 0.008 & 0.990 & 0.076 & 0.01 & $1.0 \%$ & Subsidiary risk \\
\hline N17 & Lijiagou & 0.039 & 0.930 & 0.009 & 0.07 & $7.0 \%$ & Subsidiary risk \\
\hline N18 & Zhujiaba & 0.067 & 0.973 & 0.031 & 0.027 & $2.7 \%$ & Subsidiary risk \\
\hline N19 & Dengjiatai & 0.039 & 0.920 & 0.007 & 0.08 & $8.0 \%$ & Subsidiary risk \\
\hline $\mathrm{N} 20$ & Wujiagou & 0.978 & 0.034 & 0.017 & 0.022 & $2.2 \%$ & No risk \\
\hline $\mathrm{N} 21$ & Guangou & 0.987 & 0.013 & 0.038 & 0.013 & $1.3 \%$ & No risk \\
\hline $\mathrm{N} 22$ & Meigou & 0.962 & 0.036 & 0.075 & 0.038 & $3.8 \%$ & No risk \\
\hline $\mathrm{N} 25$ & Yindongliang & 0.023 & 0.040 & 0.968 & 0.032 & $3.2 \%$ & Risk \\
\hline $\mathrm{N} 28$ & Jiangjiagou & 0.067 & 0.023 & 0.915 & 0.085 & $8.5 \%$ & Risk \\
\hline $\begin{array}{c}\text { Average } \\
\text { value }\end{array}$ & & & & & 0.051 & $5.1 \%$ & \\
\hline
\end{tabular}

\subsection{Comprehensive Evaluation of Dangerous Degree of Mine Debris Flow}

The evaluation of unknown mine debris flow risk is the final task. On the basis of the accomplishment of the training and distinction examination of artificial neural network, users can use BP network which has been trained to evaluate the dangerous degree of objective mine debris flow by comprehensive evaluation modules. At the same time, 3 output neuron values are obtained from which we can determine the different types of the mine debris flow risk (TABLE 2). 
Table 2. Intelligent evaluation result of mine debris flow Risk

\begin{tabular}{clcccc}
\hline Number & Debris flow & Neuron 1 & Neuron 2 & Neuron 3 & Results of comprehensive evaluation \\
\hline N23 & Tangjiagou & 0.995 & 0.025 & 0.012 & No risk \\
N24 & Tancaogou & 0.987 & 0.051 & 0.009 & No risk \\
N26 & Sigou & 0.012 & 0.061 & 0.985 & Risk \\
N27 & Mutonggou & 0.023 & 0.941 & 0.077 & Subsidiary risk \\
N29 & Erdaogou & 0.005 & 0.043 & 0.967 & Risk \\
N30 & Sandaogou & 0.021 & 0.020 & 0.990 & Risk \\
N31 & Sidaogou & 0.007 & 0.032 & 0.964 & Risk \\
N32 & Santaishangou & 0.027 & 0.982 & 0.045 & Subsidiary risk \\
N33 & Shangpinggou & 0.058 & 0.992 & 0.003 & Subsidiary risk \\
N34 & Hougou & 0.086 & 0.965 & 0.027 & Subsidiary risk \\
\hline
\end{tabular}

\section{Conclusion}

There are numerous factors controlling the mine debris flow risk. This paper put forward an index system of evaluating the mine debris flow risk on the basis of analyzing various examples of mine debris flow and developed the intelligent comprehensive evaluation system of the mine debris flow risk based on IGA-BP. This system absorbs a series of advantages of database application, genetic algorithm, immune algorithm and artificial neural network. It adopts the ideas of object-oriented and humanity program design and realizes the objective comprehensive evaluation of the mine debris flow risk. Before the use of software system, it requires geological professionals to observe at spot and collect related raw data of terrain, physiognomy, lithological character, structure, mine development and so on. Then set the basic data base of mine debris flow and select network training samples. The software modules will automatically realize index quantification processing, immune genetic calculation, artificial neural network training and the comprehensive evaluation of the mine debris flow risk.

\section{Acknowledgements}

Thanks to the subsidy of Shaanxi Nature Science Fund Project (SJ08-ZT08-3).

\section{References}

[1] HOU Enke, HAO Zhucheng, WANG Xiangyang, "The present state of geological environment and restoring strategy in lead-zinc mining areas in Feng Xian," Nonferrous Metals(Mining), No. 5, pp.12-14, 2001.

[2] CUI Peng, WEI Fangqiang, XIE Hong etc, "Debris flow and disaster reduction strategies in western china," Quaternary sciences, Vol. 23, No. 2, pp. 142-151. 2003.

[3] LIU xilin, TANG Chuan, "The mudslide risk evaluation," Beijing: Science publisher, 1995. 
[4] WEI yongming, XIE Youyu, WU Yongqiu, "Applications of relativity analysis method and fuzzy synthetical assessment method in classification of dangerous degree of debris flow," Natural disaster college journal, Vol. 7, No. 2, pp. 109-117, 1998.

[5] LIU Yongjiang, HU Houtian, BAI Zhiyong, "Mudslide dangerous degree the nerve network method for evaluate," The geology and Prospecting, Vol. 37, No. 2, pp.84-87, 2001.

[6] KUANG Lehong, LIU Baochen, YAO Jingcheng, "Research on Regionalization of Debris Flow Risk Degreewith Fuzzy and Extension Method," Catastrophology, Vol. 21, No.1, pp. 68-72, 2006.

[7] LIU Jinfeng, OU Guoqiang, "New opinion on debris flows hazard assessment," The geology disaster and environmental protection, Vol. 15, No. 1, pp. 5-8, 2004.

[8] XIA Yucheng, CHEN Lianwu, XUE Xi-cheng, "Geoscience information numeralization outline," Xi'an: Shaanxi Science and Technology Press, 2003.

[9] Soon Thiam Khu, "Genetic programming and its application in real-time runoff forecasting," Journal of the American Water Resources Association, Vol. 23, No. 2, pp. 439-450,2001.

[10] J G Na, "Adaptive optimization of fed-batch culture of yeast by using genetic algorithms," Bioprocess and biosystems engineering, No. 4, pp. 299-308, 2002. 